\title{
ON FREQUENCY ESTIMATION FOR PARTIALLY OBSERVED SYSTEM WITH SMALL NOISES IN STATE AND OBSERVATION EQUATIONS
}

We consider the problem of frequency estimation of the periodic signal multiplied by a Gaussian process (Ornstein-Uhlenbeck) and observed in the presence of the white Gaussian noise. We demonstrate the consistency and asymptotic normality of the maximum likelihood and Bayesian estimators in the sense of the small noise asymptotics. The model of observations is a linear nonhomogeneous partially observed system and the construction of the estimators is based on the Kalman-Bucy filtration equations. For the study of the properties of the estimators, we apply the techniques introduced by Ibragimov and Has'minskii.

Keywords: partially observed linear system, stochastic signal, frequency estimator, maximum likelihood method, Bayesian approach, characteristics of estimators, small noise asymptotic

\section{Introduction}

The problem of the estimation of the frequency (Doppler shift) of the signal is of a great importance in the fields of radioand-hydroacoustic communications and positioning, radio-andhydrolocation, radio-and-hydroacoustic positioning, etc. [1-3] The simplest model for the useful signal is the harmonic wave with the amplitude $A$ and the frequency $\vartheta_{0}$ :

$$
s(t)=A \cos \left(2 \pi \vartheta_{0} t\right)
$$

While propagating, the signal $s(t)$ can be distorted by both additive $n(t)$ and multiplicative $Y(t)$ interferences [3-6]. As the model of the additive interferences, following [1-3], we choose the white Gaussian noise (WGN) with the double-sided spectral density $\sigma_{2}$ (correlation function $\left\langle n\left(t_{1}\right) n\left(t_{2}\right)\right\rangle=\sigma^{2} \delta\left(t_{2}-t_{1}\right)$ ). For the statistical description of the multiplicative interferences, the stationary centered Gaussian random process can be often applied $[3,7]$. As a result, the mix

$$
X(t)=A \cos \left(2 \pi \vartheta_{0} t\right) Y(t)+n(t), \quad 0 \leq t \leq T
$$

is available for an observation.

We presuppose that the process $Y(t)$ satisfies a linear stochastic equation. The simplest examples are the following.

Example 1. Ornstein-Uhlenbeck (O-U) process

$\dot{Y}(t)+a Y(t)=b N(t), \quad 0 \leq t \leq T$

where $\dot{Y}(t)=d Y(t) / d t ; a>0, b>0$ are certain parameters; $N(t)$ is WGN with the unit double-sided spectral density, while the processes $n(t)$ and $N(t)$ are statistically independent. As it is well known [2], the correlation function $R(\tau)$ and the spectral density $G(\lambda)$ of the process $Y(t)$ are determined as

$R(\tau)=b^{2} \exp (-a|\tau|) / 2 a \quad G(\lambda)=b^{2} /\left(a^{2}+4 \pi^{2} \lambda^{2}\right)$

Example 2. The process $Y(t)$ described by the equation

$$
\ddot{Y}(t)+a \dot{Y}(t)+a_{0} Y(t)=b N(t), \quad 0 \leq t \leq T
$$

subject to condition $a_{1}^{2}<4 a_{0}$. For the correlation function and the spectral density of the process $Y(t)(5)$, we have [2]

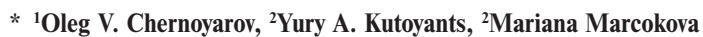

${ }^{1}$ Department of Electronics and Nanoelectronics, National Research University "Moscow Power Engineering Institute", Russia and International Laboratory of Statistics of Stochastic Processes and Quantitative Finance of Tomsk State University, Russia

${ }^{2}$ Laboratoire Manceau de Mathématiques, Le Mans University, France and International Laboratory of Statistics of Stochastic Processes and Quantitative Finance of Tomsk State University, Russia

${ }^{3}$ Department of Structural Mechanics and Applied Mathematics, University of Zilina, Slovakia

E-mail: chernoyarovov@mpei.ru
} 


$$
\begin{aligned}
& R(\tau)=\frac{b^{2}}{2 a_{0} a_{1}} \exp (-\gamma|t|)\left[\cos (\omega \tau)+\frac{\gamma}{\omega} \sin (\omega|\tau|)\right] \\
& G(\lambda)=\frac{b^{2}}{\left[a_{0}-4 \pi^{2} \lambda^{2}\right]^{2}+4 \pi^{2} a_{1}^{2} \lambda^{2}}
\end{aligned}
$$

Here $\gamma=a_{1} / 2$ and $\gamma^{2}+\omega^{2}=a_{0}$.

For simplicity of description, we presuppose that $Y(t)$ is the $\mathrm{O}-\mathrm{U}$ process and we refer to the formalism of stochastic calculus. It should be noted that all the results presented below for the O-U process can be directly extended to encompass not only the model presented in Example 2, but the much more complicated linear partially observed systems as well, though in this case the calculus and the expressions involved turn to be much more cumbersome.

Thus, we have the observation model (2) and (3) presented in the form of the two-dimensional stochastic process $\left(X_{t}, Y_{t}, 0 \leq t \leq T\right)$ satisfying the differential equations

$$
\begin{array}{ll}
d X_{t}=A \cos \left(2 \pi \vartheta_{0} t\right) Y_{t} d t+\sigma d W, & X_{0}=0 \\
d Y_{t}=-a Y_{t} d t+b d V_{t}, & Y_{0}=y_{0}
\end{array}
$$

Here $W_{t}, 0 \leq t \leq T$ and $V_{t}, 0 \leq t \leq T$ are two independent Wiener processes. The parameters $A, \sigma, a, b$ are presupposed to be already known and positive, while the parameter $\vartheta_{0} \in \Theta=(\alpha, \beta)$ is unknown and has to be estimated by the observations $X^{T}=X(T)(2)$. We have partially observed linear system, where the component $Y_{T}$ is not observable.

In $[1,3]$, the estimate is considered of the band center of random process with the fractional rational spectrum observed against Gaussian white noise; in [5], the results [1, 3] are generalized for the case when the spectral density of the random process is described by Gaussian curve. At last, in [6], the characteristics are found of the estimate of the band center of random process with free-form spectral density. And it is presupposed everywhere that the analyzed random process is stationary, while the asymptotic properties of the introduced band center estimates are examined under $T \rightarrow \infty$ only. The further extension of the specified problem is the use of non-stationary model of the useful random signal of the type (7) describing a practically important wide class of stochastic information processes and obtaining the properties of the estimate of unknown frequency parameter in wider range of asymptotics. In such context, the problem of statistical analysis of the estimate of random process band center is considered for the first time.

We now study two estimators of the parameter $\vartheta: \hat{\vartheta}_{T}$ - the maximum likelihood estimator (MLE) and $\tilde{\vartheta}_{T}$ - the Bayesian estimator (BE). Let us remind the definitions of these estimators. We designate the current value of the unknown parameter $\vartheta_{0}$ as $\vartheta$ and introduce the conditional mathematical expectation

$$
m(\vartheta, t)=E_{\vartheta}\left(Y_{t} \mid X_{s}, 0 \leq s \leq t\right), \quad 0 \leq t \leq T
$$

Then, according to [8], the likelihood ratio function can be formulated as

$$
\begin{aligned}
& V\left(\vartheta, X^{T}\right)=\exp \left[\frac{1}{\sigma^{2}} \int_{0}^{T} \cos (2 \pi \vartheta t) m(\vartheta, t) d X_{t}-\right. \\
& \left.-\frac{1}{2 \sigma^{2}} \int_{0}^{T} \cos ^{2}(2 \pi \vartheta t) m^{2}(\vartheta, t) d t\right], \vartheta \in \Theta
\end{aligned}
$$

The MLE $\hat{\vartheta}_{T}$ is determined by the relation [1-3]

$$
V\left(\hat{\vartheta}_{T}, X^{T}\right)=\sup _{\vartheta \in \Theta} V\left(\vartheta, X^{T}\right)
$$

To introduce the BE $\tilde{\vartheta}_{T}$, we presuppose that the parameter $\vartheta$ is a random variable having the continuous positive probability density $p(\theta)$, with $\alpha<\theta<\beta$ and the quadratic loss function. Then the $\mathrm{BE}$ is [1-3]

$$
\tilde{\vartheta}_{T}=\int_{\alpha}^{\beta} \theta p(\theta) V\left(\theta, X^{T}\right) d \theta / \int_{\alpha}^{\beta} p(\theta) V\left(\theta, X^{T}\right) d \theta
$$

In our research, we are interested in the asymptotic behavior of these estimators. The asymptotics providing the consistency of the estimators can refer to the following groups of conditions: a) $\sigma \rightarrow 0, b \rightarrow 0, T$ is fixed; b) $\sigma \rightarrow 0, b$ and $T$ are fixed; c) $T \rightarrow \infty$, $\sigma$ and $b$ are fixed. One should remember that the asymptotics $A \rightarrow \infty$ can be reduced to the case $\mathrm{b}$ ).

In all the three cases, the problem of the parameter estimation is a regular one, therefore, the MLE and the BE are asymptotically normal with the natural normalization by the Fisher information $I_{T}\left(\vartheta_{0}\right)[1-3]$ :

$$
\begin{aligned}
& \sqrt{I_{T}\left(\vartheta_{0}\right)}\left(\hat{\vartheta}_{T}-\vartheta_{0}\right) \Rightarrow N(0,1) \\
& \sqrt{I_{T}\left(\vartheta_{0}\right)}\left(\tilde{\vartheta}_{T}-\vartheta_{0}\right) \Rightarrow N(0,1)
\end{aligned}
$$

According to [8], for the Fisher information in Equation (12) we can write

$$
\begin{aligned}
I_{T}(\vartheta)= & \frac{A^{2}}{\sigma^{2}} \int_{0}^{T}[\cos (2 \pi \vartheta t) \dot{m}(\vartheta, t)- \\
& -2 \pi t \sin (2 \pi \vartheta t) m(\vartheta, t)]^{2} d t
\end{aligned}
$$

Here and in the sequel dot means differentiation w.r.t. $\vartheta$ and prime differentiation w.r.t. $t$.

We suggest a slightly more general model for the observations

$d X_{t}=f\left(\vartheta_{0} t\right) Y_{t} d t+\sigma d W_{t}, \quad X_{0}=0, \quad 0 \leq t \leq T$

$d Y_{t}=-a(t) Y_{t} d t+b(t) d V_{t}, \quad Y_{0}=y_{0}$

where $f(t)$ is a known smooth periodic function with the period 1 , and for the asymptotics we take the case a) when there is a small noise asymptotics in the state (15) and the observation (14) equations:

$$
\sigma=b=\varepsilon \rightarrow 0
$$


The case b) is considered in the paper [9]. The statistical problems for the partially observed linear and nonlinear systems are studied in [10]. You can find there useful references to the related statistical problems. The case $T \rightarrow \infty$ can be treated following the study of similar problems in [11], where identification of the partially observed system is considered.

\section{Auxiliary results}

The process $m(\vartheta, t), 0 \leq t \leq T$ satisfies the following equation of Kalman-Bucy filtration [8, 12]:

$$
\begin{aligned}
& d m(\vartheta, t)=-a(t) m(\vartheta, t) d t+\gamma(\vartheta, t) f(\vartheta, t) . \\
& \cdot\left[d X_{t}-f(\vartheta t) m(\vartheta, t) d t\right] / \sigma^{2}
\end{aligned}
$$

where the function $\gamma(\vartheta, t)=\mathbf{E}_{\vartheta}\left(m(\vartheta, t)-Y_{t}\right)^{2}$ is the solution for the Ricatti equation.

$$
\begin{aligned}
& \frac{\partial \gamma(\vartheta, t)}{\partial t}=-2 a(t) \gamma(\vartheta, t)- \\
& -\frac{1}{\sigma^{2}} \gamma^{2}(\vartheta, t) f^{2}(\vartheta t)+b^{2}(t) \\
& \gamma(\vartheta, t)=0
\end{aligned}
$$

Then, for the derivate $\dot{m}(\vartheta, t)$, we obtain

$$
\begin{aligned}
& d \dot{m}(\vartheta, t)=-\left[a(t)+\gamma(\vartheta, t) f^{2}(\vartheta t) / \sigma^{2}\right] \dot{m}(\vartheta, t) d t+ \\
& +h(\vartheta, t) m(\vartheta, t) d t+g(\vartheta, t)\left[d X_{t}-f(\vartheta t) m(\vartheta, t) d t\right]
\end{aligned}
$$

where $\dot{m}(\vartheta, 0)=0$ and

$$
\begin{aligned}
& g(\vartheta, t)=\left[\dot{\gamma}(\vartheta, t) f(\vartheta t)+t \gamma(\vartheta, t) f^{\prime}(\vartheta, t)\right] / \sigma^{2} \\
& h(\vartheta, t)=t \gamma(\vartheta, t) f(\vartheta t) f^{\prime}(\vartheta, t) / \sigma^{2}
\end{aligned}
$$

For the derivate $\dot{\gamma}(\vartheta, t)$, from Equation (18), we get the equation

$$
\begin{aligned}
& \frac{\partial \dot{\gamma}(\vartheta, t)}{\partial t}=-2\left[a(t)+\frac{1}{\sigma^{2}} \gamma(\vartheta, t) f^{2}(\vartheta t)\right] . \\
& \dot{\gamma}(\vartheta, t)-\frac{2 t}{\sigma^{2}} \gamma^{2}(\vartheta, t) f(\vartheta t) f^{\prime}(\vartheta t), \dot{\gamma}(\vartheta, 0)=0
\end{aligned}
$$

These equations are obtained by the formal differentiation but this derivation can be justified by the standard methods. The both functions (the Gaussian - $m(\vartheta, t)$ and the deterministic $-g(\vartheta, t)$ ) are infinitely differentiable.

Equations (19) and (21) are linear and their solutions can be found explicitly. Let us denote

$$
q(\vartheta, t)=a(t)+\gamma(\vartheta, t) f^{2}(\vartheta t) / \sigma^{2}
$$

Then we get

$$
\begin{aligned}
& \dot{m}(\vartheta, t)=\int_{0}^{t} \exp \left[-\int_{s}^{t} q(\vartheta, v) d v\right] h(\vartheta, s) m(\vartheta, s) d s+ \\
& +\int_{0}^{t} \exp \left[-\int_{s}^{t} q(\vartheta, v) d v\right] g(\vartheta, s)\left[d X_{s}-f(\vartheta s) m(\vartheta, s) d s\right] \\
& \text { and } \\
& \dot{\gamma}(\vartheta, t)=\frac{1}{\sigma^{2}} \int_{0}^{t} s \exp \left[-2 \int_{s}^{t} q(\vartheta, v) d v\right] . \\
& \gamma^{2}(\vartheta, s) f^{\prime}\left(\vartheta_{s}\right) d s
\end{aligned}
$$

\section{Main result}

While analyzing the specified asymptotics (16), we denote the MLE and the BE as $\hat{\vartheta}_{\varepsilon}$ and $\tilde{\vartheta}_{\varepsilon}$, correspondingly and we present the model of observations as follows

$d X_{t}=f\left(\vartheta_{0} t\right) Y_{t} d t+\varepsilon d W_{t}, \quad X_{0}=0, \quad 0 \leq t \leq T$

$d Y_{t}=-a(t) Y_{t} d t+b(t) \psi(\varepsilon) d V_{t}, \quad Y_{0}=y_{0} \neq 0$

Our goal is to estimate the parameter $\vartheta_{0}$ and to describe the properties of the estimators for the case when $\varepsilon \rightarrow 0, \psi(\varepsilon) \rightarrow 0$. We presuppose that the functions $a(t), b(t)$ are bounded and positive. In this study we also assume that

$\psi(\varepsilon)=\mu \varepsilon$

The case $\psi(\varepsilon) \rightarrow 0, \psi(\varepsilon) / \varepsilon \rightarrow \infty$ and the case $\psi(\varepsilon) \rightarrow 0$, $\psi(\varepsilon) / \varepsilon \rightarrow 0$ will be considered later, using the same method applied.

We introduce the function $\gamma_{*}(\vartheta, t)=\varepsilon^{-2} \gamma(\vartheta, t)$ and point out that

$$
\begin{aligned}
& d m(\vartheta, t)=-a(t) m(\vartheta, t) d t+\boldsymbol{\gamma}_{*}(\vartheta, t) f(\vartheta t) \\
& \cdot\left[d X_{t}-f(\vartheta t) m(\vartheta, t) d t\right]
\end{aligned}
$$

If the Equation (27) holds, then the function $\gamma^{*}(\vartheta, t)$ satisfies the Riccati equation

$$
\begin{aligned}
& \frac{\partial \gamma_{*}(\vartheta, t)}{\partial t}=-2 a(t) \gamma_{*}(\vartheta, t)-\gamma_{*}^{2}(\vartheta, t) f^{2}(\vartheta t)+ \\
& +\mu^{2} b^{2}(t), \quad \gamma_{*}(\vartheta, t)=0
\end{aligned}
$$

and the solution $\gamma_{*}(\vartheta, t)$ does not depend on $\varepsilon$.

We can show now that the Fisher information in this problem 
$I_{\varepsilon}(\vartheta)=\varepsilon^{-2} I_{0}(\vartheta)(1+o(1))$

where

$I_{0}(\vartheta)=\int_{0}^{T}\left[f(\vartheta t) \dot{m}_{0}(\vartheta, t)+t f^{\prime}(\vartheta t) m_{0}(\vartheta, t)\right]^{2} d t$

and $m_{0}(\vartheta, t), \dot{m}_{0}(\vartheta, t)$ are the limits of the processes $m(\vartheta, t), \dot{m}(\vartheta, t)$, correspondingly, when $\varepsilon \rightarrow 0$. It is easy to see that

$$
\begin{aligned}
m_{0}(\vartheta, t) & =y_{0} \exp \left[-\int_{0}^{t} a(s) d s\right] \\
\dot{m}(\vartheta, t) & =\int_{0}^{t} \exp \left[-\int_{s}^{t} q(\vartheta, v) d v\right] h(\vartheta, s) d s
\end{aligned}
$$

where

$h(\vartheta, t)=\gamma_{*}(\vartheta, t) f(\vartheta t)$

It is shown below (Lemma 1) that the corresponding family of measurers is locally asymptotically normal (LAN), and, therefore, we can define the lower bound (Hajek-Le Cam) for the mean square risk of any estimator $\bar{\vartheta}_{\varepsilon}$ :

$$
\lim _{\delta \rightarrow 0} \lim _{\varepsilon \rightarrow 0\left|\vartheta-\vartheta_{0}\right|<\delta} \sup \varepsilon^{-2} \mathbf{E}_{\vartheta}\left|\bar{\vartheta}_{\varepsilon}-\vartheta\right| \geq I^{-1}\left(\vartheta_{0}\right)
$$

We call an estimator $\vartheta_{\varepsilon}^{*}$ the asymptotically efficient, if for all $\vartheta_{0} \in \Theta$ we have the equality in Equation (34).

Theorem 1. Referring to the compact $K \in \Theta$, the MLE and the $\mathrm{BE}$ are uniformly consistent and asymptotically normal:

$$
\begin{aligned}
& \left(\hat{\vartheta}_{\varepsilon}-\vartheta_{0}\right) / \varepsilon \Rightarrow N\left(0, I_{0}^{-1}\left(\vartheta_{0}\right)\right) \\
& \left(\tilde{\vartheta}_{\varepsilon}-\vartheta_{0}\right) / \varepsilon \Rightarrow N\left(0, I_{0}^{-1}\left(\vartheta_{0}\right)\right)
\end{aligned}
$$

and we have the convergence of all the polynomial moments $(p>0)$ :

$\varepsilon^{-p} I_{0}^{p / 2}\left(\vartheta_{0}\right) \mathrm{E}_{\vartheta_{0}}\left|\hat{\vartheta}_{\varepsilon}-\vartheta_{0}\right|^{p} \rightarrow \mathrm{E}|\zeta|^{p}$,

$\mathcal{E}^{-p} I_{0}^{p / 2}\left(\vartheta_{0}\right) \mathbf{E}_{\vartheta_{0}}\left|\tilde{\vartheta}_{\varepsilon}-\vartheta_{0}\right|^{p} \rightarrow \mathbf{E}|\zeta|^{p}, \zeta \sim N(0,1)$

and thus both estimators are asymptotically efficient.

Proof. The proof of this theorem follows from the general results of the Theorems 3.2.1 and 3.2.2 in [13]. The conditions for these theorems are fulfilled, if we prove the following three lemmas.

Lemma 1. The normalized likelihood ratio

$$
\begin{aligned}
& Z_{\varepsilon}(u)=V\left(\vartheta_{0}+\varepsilon u, X^{T}\right) \\
& u \in U_{\varepsilon}=\left(\left(\alpha-\vartheta_{0}\right) / \varepsilon,\left(\beta-\vartheta_{0}\right) / \varepsilon\right)
\end{aligned}
$$

allows the representation

$$
Z_{\varepsilon}(u)=\exp \left\lfloor u \Delta_{\varepsilon}\left(\vartheta_{0}, X^{T}\right)-u^{2} I_{0}\left(\vartheta_{0}\right) / 2+r_{\varepsilon}\right\rfloor
$$

$$
\begin{aligned}
& \Delta_{\varepsilon}\left(\vartheta_{0}, X^{T}\right)=\int_{0}^{T}\left[f(\vartheta t) \dot{m}_{0}(\vartheta, t)+t f^{\prime}(\vartheta t) m_{0}(\vartheta, t)\right] \\
& \cdot d W \sim N\left(0, I_{0}\left(\vartheta_{0}\right)\right)
\end{aligned}
$$

Proof. Let us put that $M(\vartheta, t)=f(\vartheta t) m(\vartheta, t)$ and hence

$\dot{M}_{0}(\vartheta, t)=f(\vartheta t) \dot{m}(\vartheta, t)+t f^{\prime}(\vartheta t) m_{0}(\vartheta, t)$

As it is easy to see, we have: $M\left(\vartheta_{0}+\varepsilon u, t\right)-M\left(\vartheta_{0}, t\right)=\varepsilon u \dot{M}_{0}$ $\left(\vartheta_{0}, t\right)(1+o(1))$. Therefore,

$\ln Z_{\varepsilon}(u)=\frac{1}{\varepsilon} \int_{0}^{T}\left[M\left(\vartheta_{0}+\varepsilon u, t\right)-M\left(\vartheta_{0}, t\right)\right] d W_{t}-$

$-\frac{1}{2 \varepsilon^{2}} \int_{0}^{T}\left[M\left(\vartheta_{0}+\varepsilon u, t\right)-M\left(\vartheta_{0}, t\right)\right]^{2} d t=$

$=u \Delta_{\varepsilon}\left(\vartheta_{0}, X^{T}\right)-u^{2} I_{0}\left(\vartheta_{0}\right) / 2+r$

where $r_{\varepsilon} \rightarrow 0$.

Lemma 2. There exists a constant $C>0$, such as this

$\mathrm{E}_{\vartheta_{0}}\left|Z_{\varepsilon}^{1 / 2}\left(u_{2}\right)-Z_{\varepsilon}^{1 / 2}\left(u_{1}\right)\right|^{2} \leq C\left|u_{2}-u_{1}\right|^{2}$

Proof. Let us put

$Q_{t, \varepsilon}=\left[\frac{V\left(\vartheta_{0}+\varepsilon u_{2}, X^{t}\right)}{V\left(\vartheta_{0}+\varepsilon u_{1}, X^{t}\right)}\right]^{1 / 2}$,

$\delta_{t}=M\left(\vartheta_{0}+\varepsilon u_{2}, t\right)-M\left(\vartheta_{0}+\varepsilon u_{1}, t\right)$

Then, by applying the Ito formula, we come to the following relation

$d Q_{t, \varepsilon}=-\frac{1}{8 \varepsilon^{2}} Q_{t, \varepsilon} \delta_{t}^{2} d t+\frac{1}{2 \varepsilon} Q_{t, \varepsilon} \delta_{t} d W_{T}, Q_{0, \varepsilon}=1$

and we can write

$\mathbf{E}_{\vartheta_{0}}\left|Z_{\varepsilon}^{1 / 2}\left(u_{2}\right)-Z_{\varepsilon}^{1 / 2}\left(u_{1}\right)\right|^{2}=\mathbf{E}_{\vartheta_{1}}\left(Q_{T, \varepsilon}-1\right)^{2}=$

$=2-2 \mathbf{E}_{\vartheta_{1}} Q_{T, \varepsilon}=\frac{1}{4 \varepsilon^{2}} \int_{0}^{T} \mathbf{E}_{\vartheta_{1}} Q_{t, \varepsilon} \boldsymbol{\delta}_{1}^{2} d t \leq$

$\leq \frac{1}{8 \varepsilon^{2}} \int_{0}^{T} \mathrm{E}_{\vartheta_{1}} \delta_{1}^{2} d t+\frac{1}{8 \varepsilon^{2}} \int_{0}^{T} \mathrm{E}_{\vartheta_{2}} \delta_{1}^{2} d t \leq C\left|u_{2}-u_{1}\right|^{2}$

Here we have changed the measures: $\mathrm{E}_{\vartheta_{1}} Q_{t, \varepsilon} \delta_{1}^{2}=\mathrm{E}_{\vartheta_{2}} \delta_{1}^{2}$, where $\vartheta_{i}=\vartheta_{0}+\varepsilon u_{i}, i=1,2$, and the relations used are: $\mathrm{E}_{\vartheta_{1}} Q_{T, \varepsilon}^{2}=1$ and $2 a b \leq a^{2}+b^{2}$.

Lemma 3. There exists a constant $\kappa>0$, such as this

$\mathbf{E}_{\vartheta_{1}} Z_{\varepsilon}^{1 / 2}(u) \leq \exp \left(-\kappa u^{2}\right)$

Proof. The proof of this lemma is omitted because it is cumbersome but it follows the same steps as the proof of the Lemma 2.4 in [10].

where $r_{\varepsilon} \rightarrow 0$ and 
The properties of the normalized likelihood ratio $Z_{\varepsilon}(u)$ established in these three lemmas allows us to cite the Theorem 1.3.1 and 1.3.2 from [13] and, therefore, obtain the properties of MLE and BE, announced in the Theorem 1. Particularly, the convergence of the random process $Z_{\varepsilon}(u) \Rightarrow Z(u)$, where

$Z(u)=\exp \left\lfloor u \Delta-u^{2} I_{0}\left(\vartheta_{0}\right) / 2\right\rfloor, \Delta \sim N\left(0, I_{0}\left(\vartheta_{0}\right)\right)$

allows one to obtain the asymptotic normality of the MLE in the following way:

$P_{\vartheta_{0}}\left(\frac{\hat{\vartheta}_{\varepsilon}-\vartheta_{0}}{\varepsilon}<x\right)=P_{\vartheta_{0}}\left(\hat{\vartheta}_{\varepsilon}<\vartheta_{0}+\varepsilon x\right)=$
$=P_{\vartheta_{0}}\left[\sup _{\vartheta<\vartheta_{0}+\varepsilon x} V\left(\vartheta, X^{T}\right)>\sup _{\vartheta \geq \vartheta_{0}+\varepsilon x} V\left(\vartheta, X^{T}\right)\right]=$
$=P_{\vartheta_{0}}\left[\sup _{\vartheta<\vartheta_{0}+\varepsilon x} \frac{V\left(\vartheta, X^{T}\right)}{V\left(\vartheta_{0}, X^{T}\right)}>\sup _{\vartheta \geq \vartheta_{0}+\varepsilon x} \frac{V\left(\vartheta, X^{T}\right)}{V\left(\vartheta_{0}, X^{T}\right)}\right]=$
$=P_{\vartheta_{0}}\left[\sup _{u<x, u \in U_{\varepsilon}} Z_{\varepsilon}(u)>\sup _{u \geq x, u \in U_{\varepsilon}} Z_{\varepsilon}(u)\right] \rightarrow$
$\rightarrow P_{\vartheta_{0}}\left[\sup _{u<x} Z(u)>\sup _{u \geq x} Z(u)\right]=P_{\vartheta_{0}}(\hat{u}<x)$

Here we have changed the variable $\vartheta=\vartheta_{0}+\varepsilon u$. It is easy to see that

$\hat{u}=I_{0}^{-1}\left(\vartheta_{0}\right) \Delta \sim N\left(0, I_{0}^{-1}\left(\vartheta_{0}\right)\right)$

Thus, the asymptotic normality of the MLE is obtained. For the $\mathrm{BE}$, the corresponding limits provide us the relation

$$
\frac{\tilde{\vartheta}_{\varepsilon}-\vartheta_{0}}{\varepsilon} \Rightarrow \frac{\int_{-\infty}^{\infty} u Z(u) d u}{\int_{-\infty}^{\infty} Z(u) d u}=\frac{\Delta}{I_{0}\left(\vartheta_{0}\right)} \sim N\left(0, I_{0}^{-1}\left(\vartheta_{0}\right)\right)
$$

For the details see [13].

\section{Discussion}

It is of interest to see what would happen, if the rates $\varepsilon \rightarrow 0$, $\psi(\varepsilon) \rightarrow 0$ are different. Let us suppose that $\phi_{\varepsilon}=\varepsilon / \psi(\varepsilon) \rightarrow 0$. Then, this case would correspond to the situation when the noise in the state equation is essentially bigger than in the observation one. We are specifically concerned with the rate of the MLE convergence to the true value.

Let us consider the Riccati equation $\left[\frac{\partial \gamma_{*}(\vartheta, t)}{\partial t}+2 a(t) \gamma_{*}(\vartheta, t)\right] \phi_{\varepsilon}=$
$=-\gamma_{*}^{2}(\vartheta, t) f^{2}(\vartheta t)+b^{2}(t), \quad \gamma_{*}(\vartheta, t)=0$

where we have $\gamma_{*}(\vartheta, t)=\varepsilon^{-1} \psi^{-1}(\varepsilon) \gamma(\vartheta, t)$. Our supposition is that the functions $a(t), b(t)$ and $f(t)$ are strictly positive and bounded. It can be shown that, for any $t_{0}>0$, we have uniform convergence over $t \in\left[t_{0}, T\right]$

$\gamma_{*}^{2}(\vartheta, t)=f^{-2}(\vartheta t) b^{2}(t)[1+o(1)]$

This convergence allows us to study the asymptotics of $\dot{m}(\vartheta, t)$ and $\dot{\gamma}_{*}(\vartheta, t)$, and, therefore, to describe the mean square error $\mathrm{E}_{\vartheta}\left(\hat{\vartheta}_{\varepsilon}-\vartheta\right)^{2}$. For example, if $\psi(\varepsilon)=c>0$, then $\mathrm{E}_{\vartheta}\left(\hat{\vartheta}_{\varepsilon}-\vartheta\right)^{2}=\varepsilon I_{0}^{-1}(\vartheta)[1+o(1)]$, with a different Fisher information $I_{0}(\vartheta)$ [9]. Particularly, we show that $\varepsilon^{1 / 2}\left(\hat{\vartheta}_{\varepsilon}-\vartheta_{0}\right) \Rightarrow N\left(0, I_{0}^{-1}\left(\vartheta_{0}\right)\right)$. The general case when $\psi(\varepsilon) \rightarrow 0$ and $\phi_{\varepsilon} \rightarrow 0$ is to be treated later.

\section{Conclusion}

Thus, maximum likelihood and Bayesian estimators of the frequency of the regular periodic signal in the presence of a multiplicative interference described by the Gaussian Markov random process and the additive distortions, like the white Gaussian noise, are asymptotically normal, consistent, effective, and have the rate of convergence inversely proportional to the voltage signal-to-noise ratio. The limiting variance of the estimators is determined by the Fisher information for which the expression in explicit form can be found analytically.

The domain of applicability of the obtained theoretical results (their satisfactory concordance with the corresponding experimental data) can be evaluated on the basis of the studies presented in [1].

\section{Acknowledgement}

This study was financially supported by the Russian Science Foundation (research project No. 14-49-00079) and the Ministry of Education and Science of the Russian Federation (research project No. 2.3208.2017/4.6).

\section{References}

[1] KULIKOV, E. I., TRIFONOV A. P.: Estimation of the Signal Parameters against an Interference Background [in Russian]. Sovetskoe Radio, Moscow, 1978.

[2] PEROV, A. I.: Statistical Radio System Theory [in Russian]. Radiotekhnika, Moscow, 2003.

[3] VAN TREES, H. L.: Detection, Estimation, and Modulation Theory, Part III: Radar-Sonar Signal Processing and Gaussian Signals in Noise. Wiley, New York, 2001. 


\section{kom Nlkocie}

[4] SOBOLEV, V. S., FESHENKO, A. A.: Accuracy Cramer-Rao Bounds for a Laser Doppler Velocimeter. IEEE Transactions on Instrumentation and Measurement, 55(2), 659-665, 2006.

[5] SOBOLEV, V. S., ZHURAVEL F.A.: Quasioptimal Estimates of the Center Frequency of a Narrow-Band Normal Random Process with a Gaussian Spectrum. Optoelectronics, Instrumentation and Data Processing, 46(6), 565-570, 2010.

[6] TRIFONOV, A. P., ZAKHAROV, A. V.: Joint Estimator Characteristics of Random Radiopulse Arrival Time and Frequency. Izvestiya Vysshikh Uchebnykh Zavedenij, Radioelektronika, 45(5), 3-12, 1999.

[7] CHERNOYAROV, O. V., VACULIK, M., SHIRIKYAN, A., SALNIKOVA, A. V.: Statistical Analysis of Fast Fluctuating Random Signals with Arbitrary-Function Envelope and Unknown Parameters. Communications - Scientific Letters of the University of Zilina, 17(1A), 35-43, 2015.

[8] LIPTSER, R. S., SHIRYAYEV, A. N.: Statistics of Random Processes, I, II. (2nd edition). Springer, New York, 2001.

[9] CHERNOYAROV, O. V., KUTOYANTS, Y. A.: On Frequency Estimation for Partially Observed System with Small Noise in Observations. Submitted, 2017.

[10] KUTOYANTS, Y. A.: Identification of Dynamical Systems with Small Noise. Kluwer Academic Publisher, Dordrecht, 1994.

[11] KUTOYANTS, Y. A.: Statistical Inference for Ergodic Diffusion Processes. Springer, London, 2004.

[12] KALMAN, R. E., BUCY, R. S.: New Results in Linear Filtering and Prediction Theory. Transactions of ASME, Series D, Journal of Basic Engineering, 83(1), 95-108, 1961.

[13] IBRAGIMOV, I. A., HAS’MINSKII, R. Z.: Statistical Estimation - Asymptotic Theory. Springer, New York, 1981. 